Provided for non-commercial research and education use. Not for reproduction, distribution or commercial use.

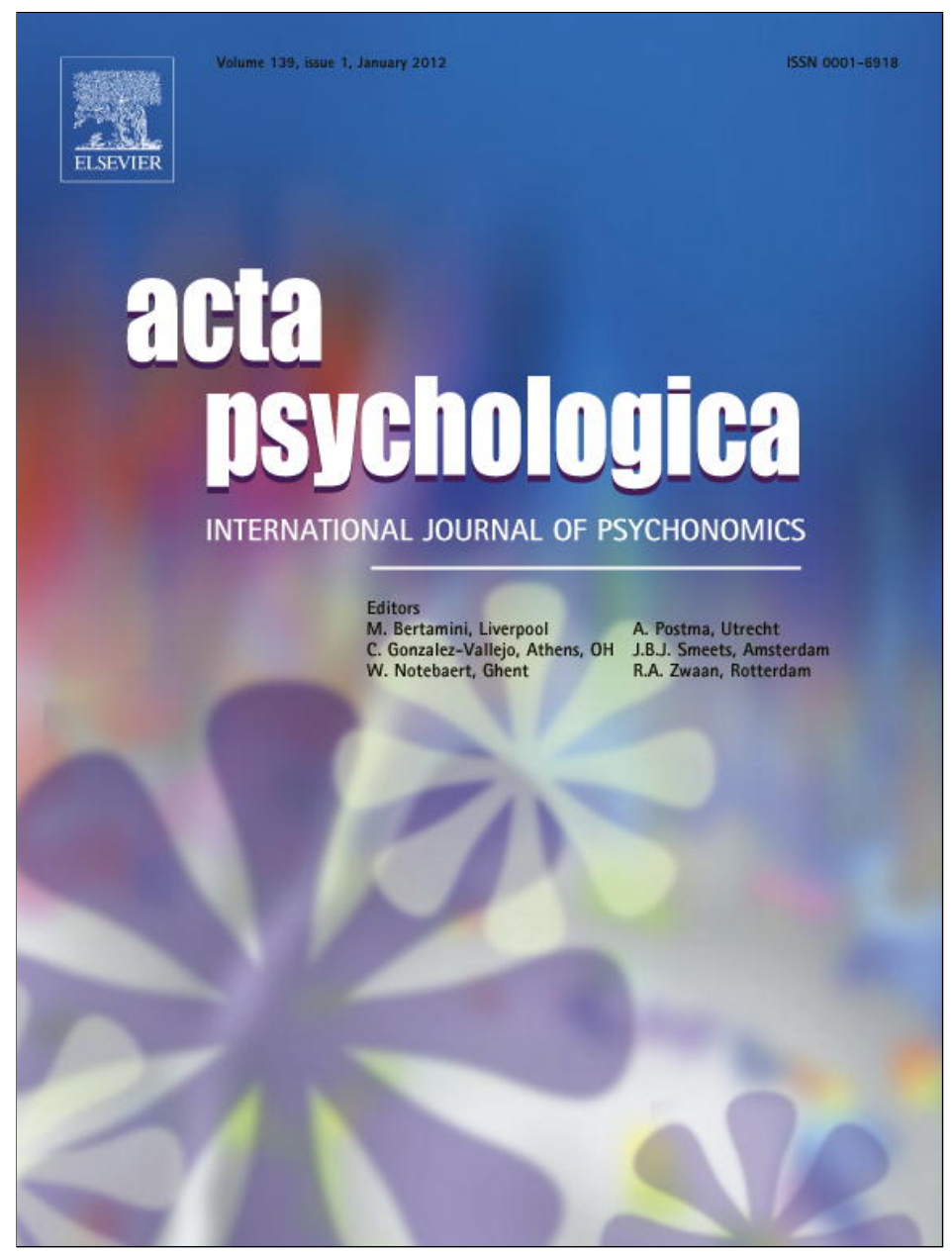

This article appeared in a journal published by Elsevier. The attached copy is furnished to the author for internal non-commercial research and education use, including for instruction at the authors institution and sharing with colleagues.

Other uses, including reproduction and distribution, or selling or licensing copies, or posting to personal, institutional or third party websites are prohibited.

In most cases authors are permitted to post their version of the article (e.g. in Word or Tex form) to their personal website or institutional repository. Authors requiring further information regarding Elsevier's archiving and manuscript policies are encouraged to visit:

http://www.elsevier.com/copyright 


\title{
Investigating the mechanisms of visually-evoked tactile sensations
}

\author{
Kirsten J. McKenzie ${ }^{\mathrm{a}, \mathrm{b}}$, Donna M. Lloyd ${ }^{\mathrm{a}}$, Richard J. Brown ${ }^{\mathrm{c}}$, Faye Plummer ${ }^{\mathrm{a}}$, Ellen Poliakoff ${ }^{\mathrm{a}, *}$ \\ a Division of Psychology, School of Psychological Sciences, Zochonis Building, University of Manchester, Brunswick Street, Manchester, M13 9PL, UK \\ b School of Psychology, Faculty of Science, University of Nottingham Malaysia Campus, Jalan Broga, 43500 Semenyih, Selangor Darul Ehsan, Malaysia

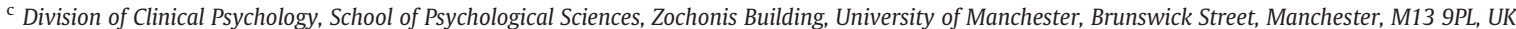

\section{A R T I C L E I N F O}

\section{Article history:}

Received 18 March 2011

Received in revised form 20 September 2011

Accepted 22 September 2011

Available online 20 October 2011

PsycINFO classification:

2320 Sensory Perception

2340 Cognitive Processes

Keywords:

Decision-making

Multisensory

Somatosensory

Touch

Vision

Perception

\begin{abstract}
A B S T R A C T
When attempting to detect a near-threshold signal, participants often incorrectly report the presence of a signal, particularly when a stimulus in a different modality is presented. Here we investigated the effect of prior experience of bimodal visuotactile stimuli on the rate of falsely reported touches in the presence of a light. In Experiment 1, participants made more false alarms in light-present than light-absent trials, despite having no experience of the experimental visuotactile pairing. This suggests that light-evoked false alarms are a consequence of an existing association, rather than one learned during the experiment. In Experiment 2, we sought to manipulate the strength of the association through prior training, using supra-threshold tactile stimuli that were given a high or low association with the light. Both groups still exhibited an increased number of false alarms during light-present trials, however, the low association group made significantly fewer false alarms across conditions, and there was no corresponding group difference in the number of tactile stimuli correctly identified. Thus, while training did not affect the boosting of the tactile signal by the visual stimulus, the low association training affected perceptual decision-making more generally, leading to a lower number of illusory touch reports, independent of the light.

(c) 2011 Elsevier B.V. All rights reserved.
\end{abstract}

\section{Introduction}

When sensory signals are presented in more than one modality simultaneously they tend to be detected faster (Hershenson, 1962), more accurately and at lower thresholds than the same signals presented individually (e.g., Frassinetti, Bolognini, \& Làdavas, 2002; Stein, London, Wilkinson, \& Price, 1996). It is generally assumed that these effects result from multisensory integration at an early processing level, whereby neural responses to multisensory stimuli are enhanced when stimuli in the different senses are spatially and temporally congruent (Meredith \& Stein, 1983). The way in which the senses are integrated seems to be driven by early sensory experience, with altered integration exhibited in animals reared in unusual sensory environments (e.g., with synchronous but spatially separate auditory and visual stimuli; Wallace \& Stein, 2007). Multisensory integration has also been reported to produce neural activity greater than the sum of responses to the two individual stimuli (Meredith \& Stein, 1983, 1986; Wallace, Meredith, \& Stein, 1998; although see Holmes, 2007, 2009a, 2009b for an alternative interpretation of these findings).

These patterns of early multisensory integration observed at a neural level have been used to explain various behavioural effects of bimodal stimuli, including the fact that task-irrelevant information

\footnotetext{
* Corresponding author. Tel.: +44161275 7333; fax: +441612752588. E-mail address: Ellen.Poliakoff@manchester.ac.uk (E. Poliakoff).
}

in one sensory modality can improve performance in another (Thorne \& Debener, 2008). The simultaneous presentation of a stimulus in one modality can, for example, increase the detection of a threshold signal in a different modality or increase the reported strength of the signal (e.g., Frassinetti et al., 2002; Lovelace, Stein, \& Wallace, 2003; Stein et al., 1996). More recently, researchers have attempted to disentangle whether the additional stimulus affects later decisional processes as well as, or instead of, earlier multisensory integration. Some multisensory effects (e.g., the effect of a visual stimulus on perceived loudness) have been observed to be independent of presentation probability and hence response bias, which is suggestive of an early effect (Odgaard, Arieh, \& Marks, 2004). Other effects may be explained fully by response bias, such as sound-induced enhancement of brightness (Odgaard, Arieh, \& Marks, 2003) and vibrotactile-induced enhancement of loudness (Yarrow, Haggard, \& Rothwell, 2008). This suggests that they result from later post-sensory decisional processes.

In studies investigating the detection of near-threshold targets during bimodal trials, researchers have used signal detection analysis (MacMillan \& Creelman, 1991) to separate sensitivity (the ability to discriminate target present from target absent trials) from bias (the tendency to say 'yes' or 'no' regardless of whether the target stimulus is presented; for this approach to visual-auditory interactions, see Frassinetti et al., 2002; Lovelace et al., 2003). In an investigation of the effect of vision on touch, Johnson, Burton, and Ro (2006) asked participants to report whether a tactile and/or visual stimulus was presented. They demonstrated that participants were more likely to detect a threshold 
tactile stimulus when it was presented alongside a supra-threshold visual stimulus, compared to when the touch was presented alone. Participants were also more likely to produce false alarms; i.e., to report touch in the absence of tactile stimulation, when the visual stimulus was present. These results were attributed to a moderate rise in tactile sensitivity and a large change in response bias. In a modified version of this task, in which participants were required to report only the presence of a tactile stimulus (the somatic signal detection task - SSDT; Lloyd, Mason, Brown, \& Poliakoff, 2008), the entirely task-irrelevant light again increased both the detection of the tactile stimulus and the number of false alarms during tactile-absent trials. Tactile sensitivity increased non-significantly in the presence of the light, but this difference has been found to be significant in follow-up studies (Brown, Brunt, Poliakoff, \& Lloyd, 2010; McKenzie, Poliakoff, Brown, \& Lloyd, 2010; Mirams, Poliakoff, Brown, \& Lloyd, 2010).

Changes in bias and the production of false alarms have typically been considered to be undesirable artefacts of the experimental procedure, confounding investigations into multisensory integration (e.g., Lovelace et al., 2003). While increases in the detection of target stimuli during bimodal trials may indeed be due to multisensory enhancement, the same cannot be said for false alarms; such responses cannot be due to multisensory integration as only a single stimulus has been presented. Prior knowledge is often required for the interpretation of incoming sensory signals (Ernst \& Bülthoff, 2004), and we would suggest that false alarm responses may represent a fundamental process in their own right, reflecting top-down effects on the perception of ambiguous stimuli. In relation to their results on the effect of touch on auditory loudness, Yarrow et al. (2008) comment "Our response bias implies an important higher-order psychological process, in this case one that is experienced as a compelling percept of increased loudness. Disregarding such an effect seems rather like throwing out the baby with the bathwater." (p. 1129). Indeed, Johnson et al. (2006) suggest that enhanced sensory detection for bimodal trials and increases in the number of false alarms may both be due to strategic sensory encoding processes, reflecting prior experience of correlated multisensory events over a person's lifetime. The idea that false alarms reflect a top-down influence on the interpretation of sensory stimuli is consistent with the finding that the tendency to false alarm is relatively stable across successive sessions, despite variability in tactile sensitivity (McKenzie et al., 2010).

In the current experiments, we studied the effect of prior experience of bimodal visuotactile stimuli on the rate of light-induced tactile sensations (false alarms) during the SSDT. Most previous experiments in this area involve a potential confound in that the bimodal trials (target stimulus plus task-irrelevant stimulus) are interspersed with the unimodal trials; consequently any increase in false alarms in the presence of the task-irrelevant stimulus may be driven by an association inherent in the experimental procedure. Therefore, in Experiment 1 we explored whether the light would increase the frequency of tactile false alarms, even when participants have had no exposure to the specific visuotactile pairing within the experiment. Finding such an effect would suggest that these false alarms are indeed a consequence of everyday multisensory experience (Johnson et al., 2006), rather than an artefact of the experimental paradigm itself. In Experiment 2, we investigated whether any existing visuotactile association could be strengthened or weakened using a training protocol and the effect that these changes would have on false alarms during the subsequent SSDT.

\section{Experiment 1}

In this experiment, we investigated whether exposure to bimodal visuotactile stimuli within the experimental session is necessary to produce an increase in false alarms on light-present trials in the SSDT, compared to light-absent trials. Rather than being a consequence of everyday experience, the previously observed effect of the light on false alarms could be produced by an association or illusory correlation built up during the experiment itself. Illusory correlations can occur when two events are perceived to be associated, despite being either unrelated or correlated to a lesser extent than reported (Chapman, 1967). If a perceived association is built up between the light and touch during the session, this could prime participants to expect (and thereby experience) touch when only the light is presented. We assessed this in Experiment 1 using a modified version of the SSDT, in which naïve participants received only unimodal stimuli during the first block of trials. Simultaneous light and tactile stimuli were then introduced in Blocks 2 and 3, before their subsequent removal in Block 4. If experience of bimodal stimuli is necessary to generate false touch reports, we would expect a minimal number of false alarms in the first block of trials, followed by a rise in blocks two and three as the visuotactile pairing is introduced. We would then expect this increase to be maintained in Block 4, despite the absence of bimodal trials. If, however, false touch reports are due to a pre-existing association, such as that formed through typical everyday multisensory experience, the effect of the light on false alarm rates would not be expected to change over the course of the experiment.

\subsection{Method}

\subsubsection{Participants}

Twenty-two naïve participants ( 4 males), aged 18 to 31 years ( mean $=21.1$ years \pm 4.41 S.D.) were included. All were right-handed (as assessed using the Edinburgh Handedness Inventory; Oldfield, 1971), had normal or corrected-to-normal vision and none reported any sensory deficits. Participants gave informed consent prior to taking part. Procedures were approved by the local ethics committee and were performed in accordance with the 1964 Declaration of Helsinki.

\subsubsection{Apparatus}

Participants sat in a light-attenuated room approximately $60 \mathrm{~cm}$ in front of a stimulus array comprised of a polystyrene wedge mounted with a red light-emitting diode (LED) $4 \mathrm{~mm}$ in diameter, and a bone conductor with $1.6 \mathrm{~cm} \times 2.4 \mathrm{~cm}$ vibrating surface (Oticon Limited, B/C 2-PIN). The participant's left index finger was affixed to the bone conductor using a double-sided adhesive pad. Tactile pulses (vibrations) were produced by sending an amplified square wave to the bone conductor, controlled by E-Prime software (Psychology Software Tools Inc., Pittsburgh, PA, USA). A PC monitor, located directly behind the stimulus array, was used to deliver instructions and cue the start of each trial with a green arrow pointing directly at the participant's finger (subtending approximately $18^{\circ} \times 7^{\circ}$ of the visual angle). White noise was played through headphones throughout the experiment to mask any distracting noise or experimentally informative sounds from the bone conductor. Participants responded with their right hand using the computer keyboard to indicate 'definitely yes', 'maybe yes', 'maybe no' or 'definitely no' for experimental trials, and 'yes' or 'no' for thresholding trials, with key order counterbalanced between participants.

\subsubsection{Procedure}

A threshold was found for each participant using the staircase method (Cornsweet, 1962), at which they reported feeling a tactile pulse in approximately $50 \%$ of trials. The starting intensity was approximately .82 metres/second (as measured by an accelerometer attached to the bone conductor). The beginning of each threshold trial was signalled by the green arrow pointing at the participant's finger for $250 \mathrm{~ms}$, followed by a stimulus period of $1020 \mathrm{~ms}$. In tactile-present trials, a $20 \mathrm{~ms}$ tactile stimulus $(100 \mathrm{~Hz})$ was delivered with a delay of $500 \mathrm{~ms}$ before and after the stimulus, and in tactileabsent trials, an empty $1020 \mathrm{~ms}$ period occurred. An onscreen prompt then instructed participants to report whether they had perceived a pulse by making a key press ('yes' or 'no'). Threshold blocks of 13 trials 
(10 tactile-present and 3 tactile-absent trials) were repeated until the stimulus intensity approached the participant's 50\% threshold. Participants were required to respond 'yes' on $40-60 \%$ of tactile-present trials at the same stimulus intensity for three consecutive blocks, before beginning the experimental trials. Feedback was not provided during thresholding or subsequent experimental blocks.

There were four blocks of 80 experimental trials. During blocks two and three, each of the four stimulus types [light (present, absent) $\times$ tactile pulse (present, absent)] was presented 20 times per block in a random order (Lloyd et al., 2008). During blocks one and four, those trials in which the light and touch were presented simultaneously were replaced with touch only (touch-present/light-absent) trials. The tactile stimulus was therefore present in $50 \%$ of trials across all four blocks, and was presented at the level established during the thresholding procedure.

The light-absent trials were identical to those described for the thresholding procedure. During the light-present trials, the LED flashed for $20 \mathrm{~ms}$ in the middle of the $1020 \mathrm{~ms}$ stimulus period, either on its own (tactile-absent), or in concert with the tactile pulse (tactilepresent). Participants were unaware of the significance of the visual stimulus and were instructed to report only the presence or absence of the tactile event. No other instructions were given, and participants were naïve as to the true purpose of the study. The total experimental time was approximately 50 min.

\subsubsection{Analysis}

In order to calculate signal detection statistics, responses were collapsed across confidence ratings: 'definitely yes' and 'maybe yes' were counted as 'yes' and 'definitely no' and 'maybe no' as 'no'. The number of hits (tactile-present trials in which participants correctly said 'yes'), false alarms (tactile-absent trials in which they incorrectly said 'yes'), misses (tactile-present trials with an incorrect 'no' response), and correct rejections (tactile-absent trials with a correct 'no' response) were counted. Hit rates and false alarm rates were then calculated, as well as the signal detection theory test statistics $d^{\prime}$ and $c$ respectively (MacMillan \& Creelman, 1991), with the log linear correction (Snodgrass \& Corwin, 1988). This provided estimates of the participants' perceptual sensitivity $\left(d^{\prime}\right)$ and tendency to report stimuli as present (i.e., response bias or $c$ ) in the light-present and light-absent conditions.

\subsection{Results}

Mean hit and false alarm rates, sensitivity indices $\left(d^{\prime}\right)$ and response criterion statistics $(c)$ as a function of block across both light-present and light-absent conditions are presented in Table 1.

False alarm rates were subjected to a 2 (Light) $\times 4$ (Block) repeated measures ANOVA. False alarms were significantly more frequent in the presence of the light $\left(\mathrm{F}_{(1,21)}=7.932, \mathrm{p}=.010\right)$, but there was no

Table 1

Mean (S.D.) hit and false-alarm rates, sensitivity indices $\left(\mathrm{d}^{\prime}\right)$ and response criterion statistics (c) across light-present and absent conditions for each block of Experiment 1.

\begin{tabular}{lllll}
\hline & \% hits & \% false alarms & $d^{\prime}$ & $c$ \\
\hline $\begin{array}{c}\text { Block 1 } \\
\text { Light-present }\end{array}$ & $\mathrm{N} / \mathrm{A}$ & $21.59(23.9)$ & $\mathrm{N} / \mathrm{A}$ & $\mathrm{N} / \mathrm{A}$ \\
$\quad$ Light-absent & $51.02(22.2)$ & $12.723(15.1)$ & $1.28(0.7)$ & $0.63(0.5)$ \\
$\begin{array}{l}\text { Block 2 } \\
\text { Light-present }\end{array}$ & $71.82(20.7)$ & $18.18(39.7)$ & $1.78(1.8)$ & $0.21(0.5)$ \\
$\quad$ Light-absent & $56.14(22.8)$ & $10.68(42.5)$ & $1.61(1.8)$ & $0.59(0.4)$ \\
Block 3 & & & & \\
$\quad$ Light-present & $72.95(17.2)$ & $17.5(37.6)$ & $1.73(1.5)$ & $0.22(0.4)$ \\
$\quad$ Light-absent & $52.5(17.2)$ & $10.45(41.9)$ & $1.42(2.1)$ & $0.64(0.4)$ \\
Block 4 & & & & \\
$\quad$ Light-present & $\mathrm{N} / \mathrm{A}(18.1)$ & $11.14(14.6)$ & $1.00(0.7)$ & $0.86(0.4)$ \\
Light-absent & $36.82(18.6)$ & &
\end{tabular}

main effect of block $\left(\mathrm{F}_{(2.077,54.805)}=1.457, \mathrm{p}=.244\right)$ and importantly there was no light $\times$ block interaction $\left(\mathrm{F}_{(3,63)}=0.255, \mathrm{p}=.857\right.$; See Fig. 1).

As we were also interested in how confident participants were in their responses we analysed the percentage of 'definitely' responses for false alarms using 2 (Light) $\times 4$ (Block) repeated measures ANOVAs. (Greenhouse-Geisser corrections have been applied for analyses in which the sphericity assumption has been violated). There was a main effect of light $\left(\mathrm{F}_{(1,21)}=9.719, \mathrm{p}=.005\right)$ indicating that participants were more confident about false alarms in the light-present condition, but there was no effect of block $\left(\mathrm{F}_{(2.168,45.528)}=.691, \mathrm{p}=.517\right)$ or block $\times$ light interaction $\left(\mathrm{F}_{(2.172,45.60)}=1.805, \mathrm{p}=.173\right)$.

As Blocks 1 and 4 did not contain trials in which the light and the touch were paired, participants were not able to produce 'hits' or 'misses' for light-present trials. Therefore, analyses of the effect of the light on hit rates, $d^{\prime}$ and $c$ were carried out for Blocks 2 and 3 only, using 2 (Light) $\times 2$ (Block) repeated measures ANOVAs. In the light trials, the hit rate was significantly higher $\left(F_{(1,21)}=33.90\right.$, $\mathrm{p}<.001)$, tactile sensitivity was significantly higher $\left(\mathrm{F}_{(1,21)}=4.505\right.$, $\mathrm{p}=.046)$ and response bias $(c)$ was significantly lower $\left(\mathrm{F}_{(1,21)}=\right.$ $29.926, \mathrm{p}<.001$ ), indicating that participants were less likely to say 'no' in the presence of the light. Most importantly, we saw no main effect of Block or any Block $\times$ Light interaction for any of the dependent variables $\left(\mathrm{Fs}_{(1,21)}<.821\right.$, all ps $\left.>.375\right)$.

Performance on light-absent trials was compared across all 4 blocks There was a significant effect of Block on hit rate $\left(\mathrm{F}_{(2.198,46.159)}=5.465\right.$, $\mathrm{p}=.006$ ). Post-hoc tests (Bonferroni corrected critical $\alpha=0.01$ ) confirmed that hit rate was significantly lower in Block 4 than all other blocks (all $\mathrm{t}_{(21)}>3.601, \mathrm{p}<.002$ ), but there were no other significant differences between Blocks (all $\mathrm{t}_{(21)}<0.802, \mathrm{p}>.432$ ). In Block 4, there was also a significantly lower $\mathrm{d}^{\prime}$ than Blocks 2 or $3\left(\mathrm{t}_{(21)}>2.950\right.$, $\mathrm{p}<.008$ ), but not Block 1 (Bonferroni corrected) and $c$ was significantly higher in Block 4 than all other Blocks $\left(\mathrm{t}_{(21)}>-2.915, \mathrm{p}<.008\right)$.

\subsection{Discussion}

The simultaneous presence of a task-irrelevant light, in close spatial proximity to a tactile event, increased reports of a near-threshold tactile stimulus. All participants displayed an increased hit rate and greater tactile sensitivity for light-present trials in comparison to

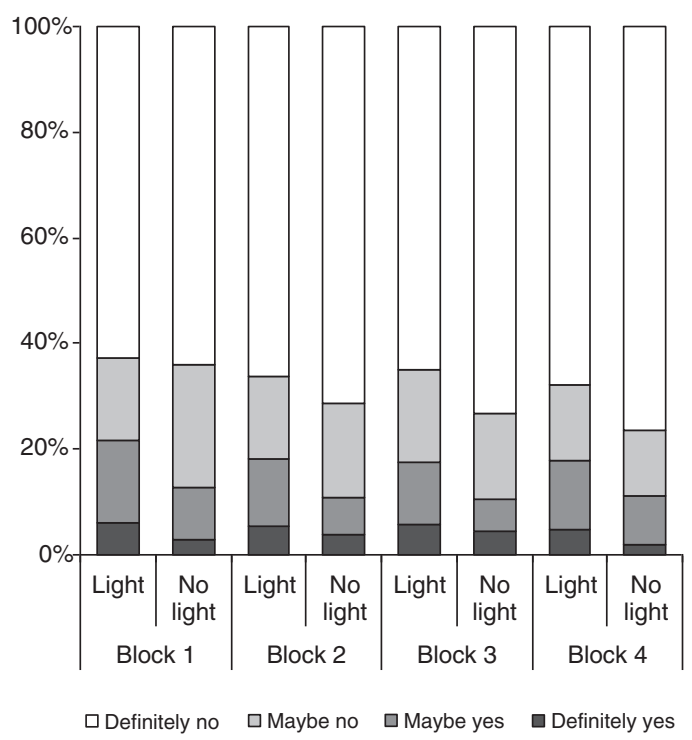

Fig. 1. Mean percentage of each type of response made in the tactile absent condition in Experiment 1 as a function of block and light condition (present, absent). It can be seen that more false alarm 'yes' responses were made in the light condition in every block. 
light-absent trials (Brown et al., 2010; McKenzie et al., 2010; Mirams et al., 2010). The presence of the light, however, also changed response criterion. There was a stronger tendency to say 'yes' in the presence of the light than without, and the number of false alarms was higher for light-present trials. This replicates previous findings showing that participants were more likely to report feeling a touch when the light was present, regardless of whether or not a touch was actually delivered (Brown et al., 2010; Lloyd et al., 2008; McKenzie et al., 2010; Mirams et al., 2010). The fact that both criterion and sensitivity were affected suggests that the increase in hits for bimodal trials was driven by both multisensory integration increasing the strength of the ambiguous tactile signal and a change in bias (hit rate was lower in the fourth block, which is likely to be due to fatigue or drift from threshold). These processes were explored further in Experiment 2.

The novel finding was that, despite not having received a paired light and touch in Block 1, participants still made more false alarms on light-present compared to light-absent trials. This differs from previous experiments in which bimodal trials are interspersed with unimodal trials throughout. Furthermore, there was no change in the pattern of false alarms when bimodal light-touch trials were included in Blocks 2 and 3 and subsequently removed in Block 4 (in which the hit rate was lower), nor did confidence ratings of the false alarms change over the course of the experiment. This indicates that prior experience of the specific light-touch pairing during the task is unnecessary for light-induced false alarms to occur, and that such exposure does not subsequently increase the number of these false alarms, at least over the time-scale that we tested. We can therefore conclude that the light-induced false alarms observed in this paradigm are not caused by an association or illusory correlation between the light and the tactile pulse, built up over the course of the experiment. Instead, it seems that these visually-induced false alarms are due to an automatic association driven by the spatial contiguity of the light and touch. This association may be the result of strategic sensory encoding processes that reflect the typical correlation between multisensory events in everyday experience (Johnson et al., 2006).

Participants often rely upon incoming visual information when making judgements about tactile stimulation, particularly when the tactile information is degraded or uncertain (Johnson et al., 2006). This uncertainty would have occurred during trials in which the incoming sensory information was not sufficient to generate an unambiguous 'yes' response. Ernst and Banks (2002) describe how perceptual judgments are based on a weighted average of the sensory signals in proportion to each signal's relative reliability in their optimal integration model (see also, Alais \& Burr, 2004). Furthermore, the dominance of a particular modality can change flexibly with alterations in signal reliability and task demands (e.g., Helbig \& Ernst, 2007; Rock \& Victor, 1964). In the current task, while the presence of the tactile signal was ambiguous, the visual information provided by the supra-threshold light flash was unequivocal. As such, the light may have been used to resolve the ambiguity of the tactile stimulus even in the absence of a tactile stimulus. If so, varying the association between the light and tactile stimulus should influence the frequency of light induced false touch reports on the SSDT. We tested this prediction in Experiment 2.

\section{Experiment 2}

To investigate the effect of the level of association between the experimental light and the tactile stimulus on light-evoked false alarms, we used a training task prior to the SSDT. A similar approach was taken by Johnson et al. (2006) in their Experiment 2 in which participants received 20 trials with a light and suprathreshold tactile stimulus prior to the main task. The effect of the light on false alarms was statistically stronger in this experiment, but false alarm rate was not compared between experiments. In their paradigm, it is also impossible to separate the association between the stimuli from the association between the light and the response (learning to respond 'both light and touch' in response to the light). To address these issues in the current experiment, participants never responded to the presence of the light itself and during the training responded to the absence of the tactile stimulus. Within the training, the light was paired with a supra-threshold tactile stimulus either frequently (75\% of trials; high association group) or infrequently (25\% of trials; low association group) and participants received trial-by-trial feedback on their performance. We predicted that following training, individuals in the high association group would make more false alarms in the presence of the light than the low association group.

This approach also afforded the opportunity to disentangle the effects of multi-sensory integration and response bias on the increased detection of tactile stimuli in bimodal trials. Manipulating the relative frequency, or probability, of stimulus presentation can produce systematic shifts in the observer's response criterion (Odgaard et al., 2003). Therefore, if we observe a between-group difference in false alarms (driven entirely by response bias), but no effect on hit rate for bimodal trials, this would suggest that purely integrational or attentional processes are involved in the increased detection of touch when accompanied by a visual stimulus. If, however, response bias is responsible for the increase in the number of hits on bimodal trials, we would predict a correlation between the effect of the light on hits (light-present minus light-absent) and the effect of the light on false alarms (light-present minus light-absent), as these would be driven by the same underlying response bias.

\subsection{Method}

\subsubsection{Participants}

Fifty right-handed naïve participants were randomly assigned to one of two experimental groups with equal numbers in each: a low association group ( 5 males, aged 18 to 35 years, mean $\pm S D=21.8 \pm$ 3.55) and a high association group ( 5 males, aged 18 to 31 years, mean $=22.2 \pm 3.58$ ). All had normal or corrected-to-normal vision and none reported any sensory deficits. An additional control group of 25 participants ( 9 males, aged 20 to 35 years, mean $=25.8 \pm$ 4.63) were included in the analysis. These participants completed the same experimental task, but received no prior training.

\subsubsection{Apparatus}

The experimental setup used was identical to that described in Experiment 1.

\subsubsection{Procedure}

Prior to thresholding, participants in the low and high association groups were required to carry out a training phase consisting of two blocks of 80 trials, using supra-threshold tactile stimuli. The intensity of these stimuli was approximately 2.5 times the intensity of the stimulus used at the beginning of the staircase thresholding procedure (1.96 metres/second). In order to minimise the similarity between the training and experimental tasks (and thereby prevent the purpose of the training phase from being identified), participants were asked to report when they did not feel a pulse, and feedback was provided on every trial.

Participants in the low association group received three times as many light alone (light-present/tactile-absent) trials as those in the high group, and the high group received three times as many bimodal (light-present/tactile-present) trials as those in the low group. Both groups received exactly the same number of tactile pulses during the training phase (see Table 2). Following the training phase, participants were given a two minute break prior to commencing the thresholding procedure (as described in Experiment 1). As the amount of time spent determining a tactile threshold varied between 
individuals (thereby affecting the time between the initial training period and experimental trials), the number of thresholding blocks was included as a covariate for analysis.

All participants completed four blocks of 80 SSDT trials, with a further thresholding procedure carried out after the first two blocks to maintain an average hit rate of $50 \%$ in light-absent trials. Each of the four stimulus conditions [light (present, absent) $\times$ tactile pulse (present, absent)] was presented 20 times per block in a random order and participants responded 'definitely yes', 'maybe yes', 'maybe no' or 'definitely no' to indicate whether they had felt a tactile pulse.

Upon completion of the experimental session, participants in the low and high association groups were asked whether they thought that the proportion of bimodal trials differed between the training and the SSDT task. Those participants giving an affirmative response were also asked to indicate the direction this difference was in, i.e., if they had received more or less bimodal trials in the training phase. In total, the session lasted approximately $1.25 \mathrm{~h}$.

\subsection{Results}

Awareness of the different contingencies between training and experimental trials varied between participants. In the high group, $8 / 25$ participants reported that they were aware of receiving more bimodal trials during the training phase and in the low group 6/25 participants were aware that the training had contained fewer bimodal trials. Therefore, awareness of the association was included as a covariate in subsequent analyses, but was not found to reach significance for any of the dependent variables tested (all $\mathrm{F}_{(1,47)}<0.491$, $\mathrm{p}>.228$ ). The two groups did not differ in the number of blocks that they required to reach a stable threshold at the start $\left(t_{(48)}=0.489\right.$, $\mathrm{p}=.629)$ or midpoint $\left(t_{(48)}=0.2, \mathrm{p}=.843\right)$ of the SSDT task, nor did the intensity level differ at either time point ( start $t_{(48)}=.281$, $\mathrm{p}=.781$; midpoint $t_{(48)}=.658, \mathrm{p}=.517$ )

Data was collapsed across confidence ratings and hit rate, false alarm rate, sensitivity $\left(d^{\prime}\right)$ and decision criterion $(c)$ were calculated for each participant in the light-present and light-absent conditions (Table 3). Mixed design ANOVAs were performed on each dependent variable [Group (high, low $) \times$ Light $($ present, absent $) \times \operatorname{Block}(1,2,3,4)$ ] Since there was no main effect of Block, nor any interactions with this factor for any dependent variable (all $\mathrm{F}_{(3,141)}<1.520, \mathrm{p}>.212$ ), the data were collapsed across Block for subsequent analysis.

\subsubsection{False alarms}

The presence of the light was found to increase the number of false alarms overall $\left(\mathrm{F}_{(1,47)}=13.722, \mathrm{p}=.001\right)$. There was also a main effect of group with the low group producing fewer false alarms than the high group $\left(\mathrm{F}_{(1,47)}=4.862, \mathrm{p}=.032\right)$. However, these two variables did not interact $\left(\mathrm{F}_{(1,47)}=1.971, \mathrm{p}=.167\right)$, indicating that participants in the low group made fewer false alarms than participants in the high group in both the presence and absence of the light (see Fig. 3).

In order to determine if this difference between groups was due to an increase in false alarms after training for the high group or a reduction in false alarms for the low group, we compared the results to a

\section{Table 2}

Number of trials in each condition for one block, as a function of association group for Experiment 2

\begin{tabular}{llll}
\hline Light & Touch & $\begin{array}{l}\text { Low } \\
\text { association }\end{array}$ & $\begin{array}{l}\text { High } \\
\text { association }\end{array}$ \\
\hline Absent & Absent & 10 & 30 \\
Absent & Present & 30 & 10 \\
Present & Absent & 30 & 10 \\
Present & Present & 10 & 30 \\
\hline
\end{tabular}

Table 3

Mean (S.D.) hit and false alarm rates, sensitivity indices ( $\left.d^{\prime}\right)$ and decision criterion statistics (c) for the low and high association groups in Experiment 2, collapsed across certainty.

\begin{tabular}{lllll}
\hline & \% hits & \% false alarms & $d^{\prime}$ & $c$ \\
\hline High & & & & \\
$\quad$ Light-present & $59.43(14.7)$ & $17.5(15.7)$ & $1.31(0.7)$ & $-0.41(0.4)$ \\
$\quad$ Light-absent & $42.94(11.4)$ & $11.66(11.7)$ & $1.17(0.6)$ & $-0.77(0.3)$ \\
Low & & & & \\
$\quad$ Light-present & $63.9(15.2)$ & $9(9.4)$ & $1.91(0.8)$ & $-0.58(0.3)$ \\
$\quad$ Light-absent & $48.85(15)$ & $6.35(5.8)$ & $1.61(0.7)$ & $-0.83(0.3)$ \\
\hline
\end{tabular}

control group who had performed the same task with no training phase. A $2 \times 2$ Group (low, control) $\times$ Light (present, absent) mixed design ANOVA revealed a main effect of group $\left(F_{(1,48)}=7.889\right.$, $\mathrm{p}=.007$ ) with the low group making significantly fewer false alarms than the control group, but there was no light $\times$ group interaction $\left(\mathrm{F}_{(1,48)}=0.037, \mathrm{p}=.849\right)$. In contrast, a $2 \times 2$ Group (high, control $) \times$ Light (present, absent) mixed design ANOVA found no effect of group $\left(\mathrm{F}_{(1,48)}=0.122, \mathrm{p}=.729\right)$ nor any light $\times$ group interaction $\left(\mathrm{F}_{(1,48)}=\right.$ $1.429, \mathrm{p}=.238$; see Fig. 2). Rather than increasing the number of false alarms made by the high association group, therefore, the training phase seems to have decreased the number of false alarms made by the low association group relative to the control group.

Since group had an effect on false alarms in both light-present and light-absent conditions, post-hoc tests were used to examine the correlation between the percentage of false alarms that individuals made in the light-present and light-absent conditions. They were highly correlated for both the high group $(r=.843, \mathrm{p}<.001)$ and the low group $(r=.614, \mathrm{p}=.001$; Fig. $3 \mathrm{~A})$.

\subsubsection{Hits}

A main effect of light was found for hit rate, with an increase in hits when the light was present $\left(\mathrm{F}_{(1,47)}=94.403, \mathrm{p}<.001\right)$, but no main effect of group was observed $\left(\mathrm{F}_{(1,47)}=2.255, \mathrm{p}=.140\right)$. To test whether the increase in hit rate in the presence of the light (hit rate light-present minus hit rate light-absent) was related to the lightrelated increase in false alarm rate (false alarm rate light-present minus false alarm rate light-absent), correlations were carried out between these two measures. They were found to be non-significant for both groups (both $r<.220, \mathrm{p}>.291$; Fig. 3B).

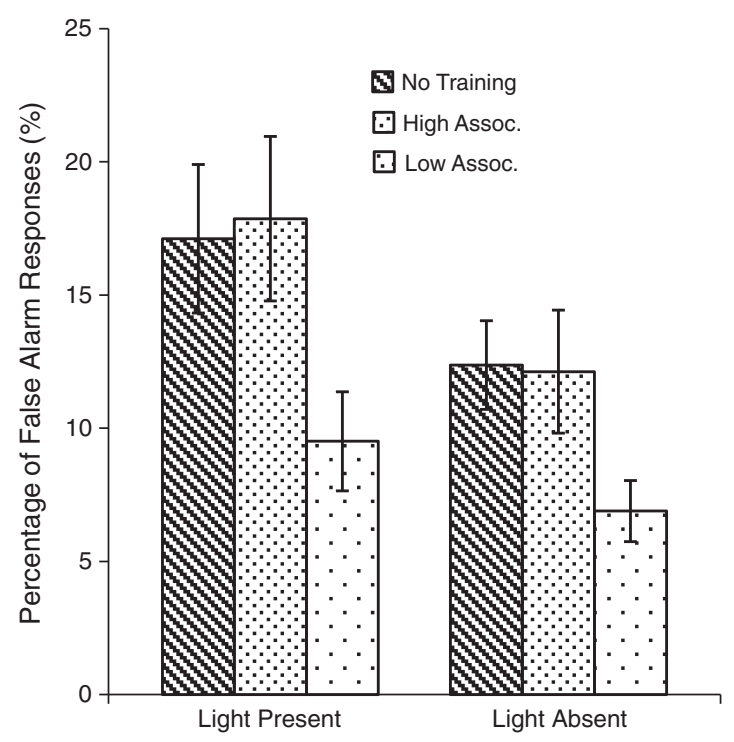

Fig. 2. Mean ( \pm 1 S.E.) percentage of False Alarms in Experiment 2 as a function of training (High association, Low association, No training) and light condition. 
A

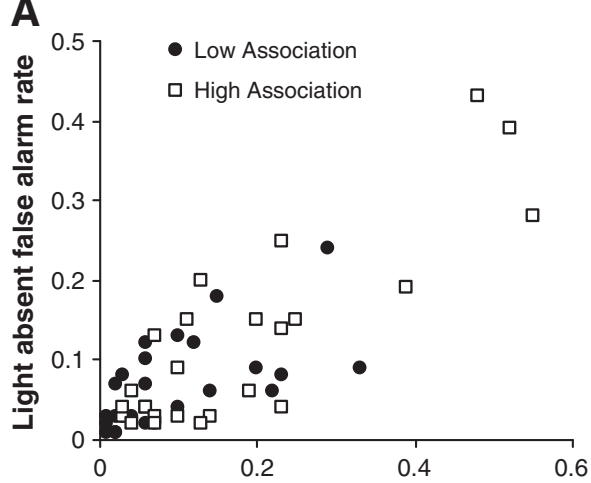

Light present false alarm rate

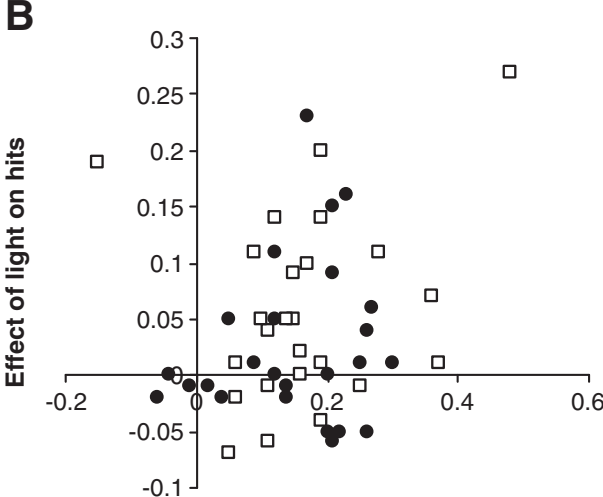

Effect of light on false alarms

Fig. 3. Correlations between the rate of false alarms in the light present and light absent conditions (panel A) and between the effect of the light on hit rate (light-present minus light-absent hit rate) and the effect of the light on the false alarm rate (panel B) for the low (black circles) and high (white squares) association groups in Experiment 2.

\subsubsection{Tactile sensitivity and response criterion}

Sensitivity to touch $\left(d^{\prime}\right)$ was found to be significantly higher in the light present compared to light absent condition $\left(\mathrm{F}_{(1,47)}=10.442\right.$, $\mathrm{p}=.002$ ) and tactile sensitivity was significantly higher in the low association group compared to the high association group $\left(\mathrm{F}_{(1,47)}=\right.$ $7.605, \mathrm{p}=.008)$. There was, however, no group $\times$ light interaction $\left(F_{(1,47)}=1.252, p=.269\right)$. There was also a highly significant effect of light on response criterion (c) with participants more likely to report the presence of a touch when the light was present than absent, regardless of whether or not a touch was actually delivered $\left(\mathrm{F}_{(1,47)}=\right.$ $59.454, \mathrm{p}<.0005)$. However, there was no effect of group $\left(\mathrm{F}_{(1,47)}=\right.$ $1.491, \mathrm{p}=.228)$ or group $\times$ light interaction $\left(\mathrm{F}_{(1,47)}=1.894, \mathrm{p}=.175\right)$.

\subsubsection{Confidence ratings}

As we were also interested in any effect that training may have had upon how confident participants were in their responses (Fig. 4) we analysed the percentage of 'definitely' responses for each response type. It was found that participants were significantly more confident about hits in the presence of the light $\left(\mathrm{F}_{(1,47)}=63.961, \mathrm{p}<.0005\right)$, and there was a trend towards a main effect of group $\left(F_{(1,47)}=3.567\right.$, $\mathrm{p}=.065)$, as well as a marginal trend towards a light $\times$ group interaction $\left(\mathrm{F}_{(1,47)}=3.146, \mathrm{p}=.083\right)$.

Confidence in false alarms was also higher when the light was present $\left(F_{(1,47)}=4.236, p=.045\right)$, but there was no main effect of group $\left(F_{(1,47)}=0.662, p=.420\right)$ nor interaction $\left(F_{(1,47)}=0.413\right.$, $\mathrm{p}=.523$ ). Conversely, the presence of the light made participants less confident in misses $\left(\mathrm{F}_{(1,47)}=9.952, \mathrm{p}=.004\right)$, although there was no difference between the two groups $\left(F_{(1,47)}=0.133\right.$, $\mathrm{p}=.717)$ nor any interaction $\left(\mathrm{F}_{(1,47)}=.878, \mathrm{p}=.354\right)$. Confidence in correct rejections was also significantly lower in the presence of the light $(\mathrm{F}(1,47)=8.525, \mathrm{p}=.005)$ and confidence in correct rejections was greater in the low group $\left(\mathrm{F}_{(1,47)}=5.085, \mathrm{p}=.029\right)$, but there was no light $\times$ group interaction $\left(\mathrm{F}_{(1,47)}=0.121, \mathrm{p}=.730\right)$ (Fig. 4).

\subsection{Discussion}

The overall effect of the light in Experiment 2 is consistent with the results of Experiment 1 and previous studies (Brown et al., 2010; McKenzie et al., 2010; Mirams et al., 2010). All participants displayed both an increased hit rate and greater tactile sensitivity for lightpresent trials compared to light-absent trials, regardless of group designation. The presence of the light also increased the tendency to say 'yes': the mean response criterion was lower, and false alarm rate higher, for light-present compared to light-absent trials. This was also reflected in how confident participants were in their responses, with confidence in reporting hits and false alarms ('yes' responses) being higher in light- present trials, and confidence in miss and correct rejection responses ('no' responses) being lower when a light was presented.

Importantly, differences on the SSDT were observed between the two training groups. Although both groups made more false alarms in the light-present condition, the low association group made significantly fewer false alarms independent of light condition. Comparison with the control group indicated that this group difference was due to false alarms being reduced in the low association group, rather than increased in the high association group. The reduction in false alarm rate in the low group, without a corresponding reduction in hits, led to a higher sensitivity being observed in this group. Confidence levels for false alarms did not differ between the groups, however, although the low association group exhibited greater confidence in making correct rejections. Importantly, the two groups did not differ significantly in the number of thresholding blocks, nor the intensity at which threshold was reached, suggesting that the training did not exert its influence via changes in the thresholds obtained.

The observed between-group differences raise several issues. Firstly, why did the training lead to the predicted decrease in the number of false alarms in the presence of light in the low association group, but no increase in false alarms in the high association group? A

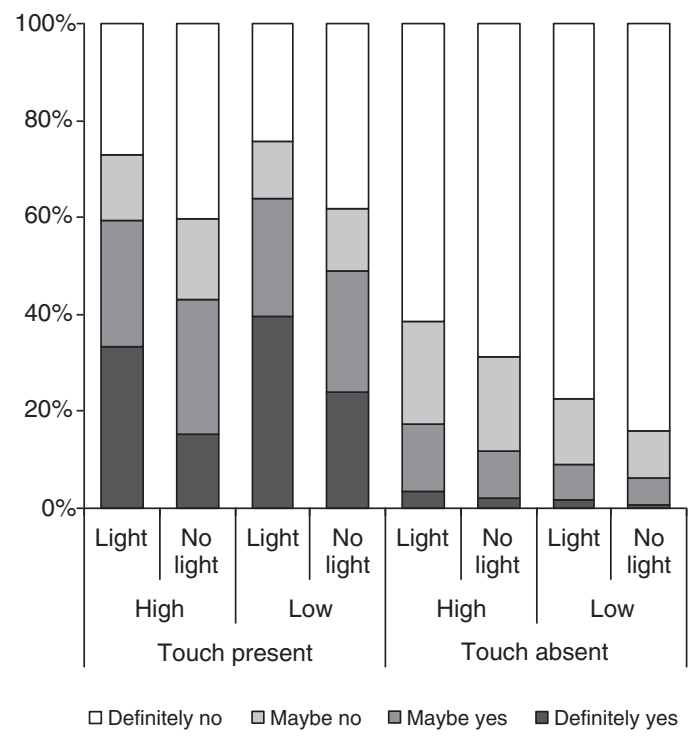

Fig. 4. Mean percentage of each type of response made in the touch present and absent conditions as a function of training (High association, Low association) and light condition (present, absent) in Experiment 2. 
similar result was reported by Odgaard et al. (2003) when investigating crossmodal enhancement of supra-threshold visual stimuli presented with concurrent white noise bursts. They found that reducing the probability of the bimodal stimulus presentation from .50 to .25 eliminated the crossmodal enhancement of ratings, whereas enhancement was still observed when the probability was increased to .75. It should be noted, however, that these probabilities occurred during the experiment itself rather than in a prior training block. The fact that false alarm rate was not increased by the short training period in Experiment 2 fits with the notion that the effect of the light is reliant upon a life-long association between visual and tactile stimuli in close proximity (Johnson et al., 2006). If this is the case, it is unsurprising if this learning/training is already at ceiling.

Secondly, we predicted that group differences would emerge only for false alarms in the light-present condition, but the low group exhibited a lower false alarm rate in the presence and absence of the light. This suggests that similar perceptual decision-making processes occurred both with and without the additional light; indeed, false alarm rate was highly correlated between light-present and light-absent conditions. Thus, changing the way that participants responded to the light-present trials had a knock-on effect on the light-absent trials (see General discussion for possible mechanisms).

A third issue to be considered is why the light still exerted an effect on false alarms in the low group. Again, this may be due to learning over a lifetime. It would be surprising if this could be completely abolished by a short training period, and it should also be noted that participants in the low association group still experienced the light paired with supra-threshold tactile stimuli in $25 \%$ of the training trials. Indeed, this is in keeping with the findings in Block 1 of Experiment 1, where false alarms increased in the presence of the light despite participants never having experienced the visual and tactile stimuli together.

Finally, as mentioned in the Introduction, this experiment allowed us to examine the effect of changing visual-tactile associations on the hit rate for bimodal visuotactile stimuli. We observed a reduction in false alarm rate in the low association group, without a corresponding drop in hit rate. Regardless of the level of association, participants displayed an increased tactile sensitivity when the light was presented as well as an increased hit rate. This would imply that multisensory integration, rather than simply bias, exerted an effect on bimodal trials. Furthermore, the increase in hit rate from the light-absent to the lightpresent condition did not correlate with the increase in false alarm rate in the presence of the light, suggesting that the light may have had a differential effect in the presence versus absence of a tactile stimulus.

\section{General discussion}

In the current work, we investigated whether experience of bimodal visuotactile stimuli is necessary to produce light-induced tactile false alarms. Experiment 1 demonstrated that experience of the paired visuotactile stimuli received within the experiment was not required in order to see an increase in the level of false alarms in the presence of a light. Furthermore, false alarm rate did not change after subsequent exposure to bimodal stimuli. This indicates that, rather than being an artefact of the experimental session, false alarms appear to be an emergent property of the system; that is, a consequence of everyday multisensory experience (Johnson et al., 2006). The robustness of this effect is further emphasised by the fact that the light exerted an effect on false alarms for both training groups in Experiment 2.

Examination of the confidence ratings across the experiments reveals more about the nature of the light-evoked false alarms. Over $20 \%$ of these false alarms were 'definitely' responses, despite the option for participants to report that they were uncertain of their response. This suggests that false alarms are not merely the product of guessing in response to an ambiguous stimulus (cf., Roediger \& McDermott, 1999). The notion that illusory false reports of touch correspond to subjective experience (cf., Yarrow et al., 2008) fits with the findings that false alarm rate is relatively stable across testing sessions (McKenzie et al., 2010) and that people who report experiencing higher numbers of somatoform dissociative symptoms (such as numbness or dizziness) in their daily lives have a higher false alarm rate (Brown et al., 2010).

As described above, participants may rely on the unequivocal light stimulus to make perceptual decisions about touch under conditions of uncertainty (cf., Ernst \& Banks, 2002). Participants seem to operate under the assumption that spatially and temporally coincident multisensory signals are produced by the same underlying event. Therefore, even the unimodal light component can be sufficient to suggest that the entire stimulus is present. This is not multisensory integration per se, since only a single stimulus is presented. However, there are neurophysiological data indicating that following exposure to a multisensory stimulus, subsequent presentations of an individual unimodal component can produce the same multisensory response, in both non-human primates (Zhou \& Fuster, 1997) and humans (Lehmann \& Murray, 2005; Murray, Foxe, \& Wylie, 2005).

Having demonstrated that false alarms were more likely in the presence of a light, even with no experience of these visuotactile stimuli, we investigated the effects of prior training with suprathreshold tactile and visual stimuli in Experiment 2. Training with strongly associated tactile and visual stimuli did not increase the number of false alarms, but training with weakly associated stimuli reduced the number of false alarms. Interestingly, this did not produce a corresponding effect on hit rate. This null effect of training on hit rate suggests that rather than being driven by later decisional processes, there was a cross-modal enhancement of bimodal visuotactile stimuli at an early processing stage; that is, the incoming tactile signal was boosted by the concurrent light (Pasalar, Ro, \& Beauchamp, 2010; see Introduction) or the light increased attention to the tactile stimulus (Lloyd et al., 2008). Indeed, a large number of participants spontaneously reported that the tactile pulse felt subjectively stronger on light-present trials.

However, in the absence of tactile stimulation, we predicted that having been exposed to a stronger association between the light and touch, the high group would make more false alarms in the presences of the light, and vice versa for the low group. Unexpectedly, however, the training led to a reduction in the number of false alarms that the low group made in both the presence and absence of the light. This is consistent with the light exerting an effect at a more general level of decision-making. de Lafuente and Romo $(2005,2006)$ have observed that activity in the medial prefrontal cortex of nonhuman primates correlates strongly with subjective experience, while activity earlier in the somatosensory processing stream relates to stimulus attributes. The timing of activity indicated that both feed-forward and feed-back mechanisms were involved in this perceptual decision making. In the current task, the reduction in false alarms led to an improvement in sensitivity in the low association group independent of the presence of the light. It seems that these participants became more 'stimulus-driven' in their perceptual decision making, relying less on top-down information from the light and their own expectations. One possible mechanism is that the degree of activation of the tactile pulse in the participant's memory determines their level of false alarms (cf., Brown, 2004); i.e., how readily this representation is re-activated either by the light or by expectations. Thus, the training may have led to a reduction in the level of activation in the low association group.

There were also limits to what training could achieve. False alarms were not increased in the high group and were still increased in the presence of the light in the low group. As discussed above, we suggest that a life-long association may already be at ceiling and difficult (or impossible) to abolish over a short training session. Future research could explore the effects of longer training periods, as well as the 
duration of the training effects. It also seemed to be critical that the training involved unambiguous supra-threshold tactile stimuli with explicit feedback. In contrast, changes in presentation probability within the SSDT itself did not have an effect in Experiment 1, nor did the 50\% probability experienced over the course of the SSDT 'undo' the association training in Experiment 2. In these cases ambiguous tactile stimuli were presented with no feedback, which may have prevented learning from occurring. Indeed, when questioned at the end of Experiment 2, the majority of participants did not report being aware of the difference in association between the training and experimental phases.

In conclusion, we have shown that experience of a paired visuotactile stimulus is not necessary to produce light-induced tactile false alarms, suggesting that this effect is a consequence of a lifetime's association between vision and touch. However, prior exposure to a weak association between these spatially congruent visuotactile stimuli did produce a general reduction in how often people reported touch in the absence of a tactile stimulus. Both effects emphasise the role of processing outside of the primary sensory modality, during the perception of ambiguous events.

\section{Acknowledgements}

This work was supported by a grant from The Leverhulme Trust [F/00 120/BF].

\section{References}

Alais, D., \& Burr, D. (2004). The ventriloquist effect results from near-optimal bimodal integration. Current Biology, 14, 257-262.

Brown, R. J. (2004). The psychological mechanisms of medically unexplained symptoms: An integrative conceptual model. Psychological Bulletin, 130, 793-812.

Brown, R. J., Brunt, N., Poliakoff, E. \& Lloyd, D. (2010). Illusory touch and tactile perception in somatoform dissociators. Journal of Psychosomatic Research, 69, 241-248.

Chapman, L. J. (1967). Illusory correlation in observational report. Journal of Verbal Learning and Verbal Behavior, 6, 151-155.

Cornsweet, T. N. (1962). The staircase-Method in psychophysics. The American Journal of Psychology, 75, 485-491.

de Lafuente, V., \& Romo, R. (2005). Neural correlate of subjective sensory experience. Nature Neuroscience, 8, 1698-1703.

de Lafuente, V., \& Romo, R. (2006). Neural correlate of subjective sensory experience gradually builds up across cortical areas. Proceedings of the National Academy of Sciences, 103, 14266-14271.

Ernst, M. O., \& Banks, M. S. (2002). Humans integrate visual and haptic information in a statistically optimal fashion. Nature, 415, 429-433.

Ernst, M. O., \& Bülthoff, H. H. (2004). Merging the senses into a robust percept. Trends in Cognitive Sciences, 8, 162-169.

Frassinetti, F., Bolognini, N., \& Làvadas, E. (2002). Enhancement of visual perception by cross-modal visuo-auditory interaction. Experimental Brain Research, 147, 332-343.

Helbig, H. B., \& Ernst, M. O. (2007). Optimal integration of shape information from vision and touch. Experimental Brain Research, 179(4), 595-606.

Hershenson, M. (1962). Reaction time as a measure of intersensory facilitation. Journal of Experimental Psychology, 63, 289-293.

Holmes, N. P. (2007). The law of inverse effectiveness in neurons and behaviour: Multisensory integration versus normal variability. Neuropsychologia, 45, 3340-3345.
Holmes, N. P. (2009). The principle of inverse effectiveness in multisensory integration: Some statistical considerations. Brain Topography, 2, 168-176.

Holmes, N. P. (2009). Inverse effectiveness, multisensory integration, and the bodily self: Some statistical considerations. Consciousness and Cognition, 18, 762-765.

Johnson, R. M., Burton, P. C., \& Ro, T. (2006). Visually induced feelings of touch. Brain Research, 1073-1074, 398-406.

Lehmann, S., \& Murray, M. M. (2005). The role of multisensory memories in unisensory object discrimination. Cognitive Brain Research, 24, 326-334.

Lloyd, D. M., Mason, L., Brown, R. J., \& Poliakoff, E. (2008). Development of a paradigm for measuring somatic disturbance in clinical populations with medically unexplained symptoms. Journal of Psychosomatic Research, 64, 21-24.

Lovelace, C. T., Stein, B. E., \& Wallace, M. T. (2003). An irrelevant light enhances auditory detection in humans: a psychophysical analysis of multisensory integration in stimulus detection. Cognitive Brain Research, 17, 447-453.

MacMillan, N. A., \& Creelman, C. D. (1991). Detection theory: A user's guide. New York: Cambridge University Press.

McKenzie, K. J., Poliakoff, E., Brown, R. J., \& Lloyd, D. M. (2010). Now you feel it, now you don't: How robust is the phenomenon of illusory tactile experience? Perception, 39 839-850.

Meredith, M. A., \& Stein, B. E. (1983). Interactions among converging sensory inputs in the superior colliculus. Science, 221, 389-391.

Meredith, M. A., \& Stein, B. E. (1986). Visual, auditory, and somatosensory convergence on cells in superior colliculus results in multisensory integration. Journal of Neurophysiology, 56, 640-662.

Mirams, L., Poliakoff, E., Brown, R. J., \& Lloyd, D. M. (2010). Vision of the body increases somatic interference on the somatic signal detection task. Experimental Brain Research, 202, 787-794

Murray, M. M., Foxe, J. J., \& Wylie, G. R. (2005). The brain uses single-trial multisensory memories to discriminate without awareness. Neurolmage, 27, 473-478.

Odgaard, E. C., Arieh, Y., \& Marks, L. E. (2003). Cross-modal enhancement of perceived brightness: Sensory interaction versus response bias. Perception \& Psychophysics, $65,123-132$.

Odgaard, E. C., Arieh, Y., \& Marks, L. E. (2004). Brighter noise: Sensory enhancement of perceived loudness by concurrent visual stimulation. Cognitive, Affective, \& Behavioral Neuroscience, 4, 127-132.

Oldfield, R. (1971). The assessment of handedness: The Edinburgh inventory. Neuropsychologia, 9, 97-111.

Pasalar, S., Ro, T., \& Beauchamp, M. S. (2010). TMS of posterior parietal cortex disrupts tactile multisensory integration. The European Journal of Neuroscience, 31 $1783-1790$

Rock, I., \& Victor, J. (1964). Vision and touch: An experimentally created conflict between the senses. Science, 143, 594-596.

Roediger, H. L., \& McDermott, K. B. (1999). False alarms about false memories. Psychological Review, 106, 406-410.

Snodgrass, J. G., \& Corwin, J. (1988). Pragmatics of measuring recognition memory: Applications to dementia and amnesia. Journal of Experimental Psychology. General $117,34-50$

Stein, B. E., London, N., Wilkinson, L. K., \& Price, D. D. (1996). Enhancement of perceived visual intensity by auditory stimuli: A psychophysical analysis. Journal of Cognitive Neuroscience, 8, 497-506.

Thorne, J. D., \& Debener, S. (2008). Irrelevant visual stimuli improve auditory task performance. NeuroReport, 19, 553-557.

Wallace, M. T., Meredith, M. A., \& Stein, B. E. (1998). Multisensory integration in the superior colliculus of the alert cat. Journal of Neurophysiology, 80, 1006-1010.

Wallace, M. T., \& Stein, B. E. (2007). Early experience determines how the senses will interact. Journal of Neurophysiology, 97, 921-926.

Yarrow, K., Haggard, P., \& Rothwell, J. C. (2008). Vibrotactile-auditory interactions are post-perceptual. Perception, 37, 1114-1130.

Zhou, Y. D., \& Fuster, J. M. (1997). Neuronal activity of somatosensory cortex in a cross-modal (visuo-haptic) memory task. Experimental Brain Research, 116 551-555. 